\title{
Electric and Magnetic Screening Masses around the Deconfinement Transition
}

\author{
Attilio Cucchieri ${ }^{a b}$, Tereza Mendes ${ }^{* a}$ \\ ${ }^{a}$ Instituto de Física de São Carlos, Universidade de São Paulo, \\ Caixa Postal 369, 13560-970 São Carlos, SP, Brazil \\ ${ }^{b}$ Ghent University, Department of Physics and Astronomy, \\ Krijgslaan 281-S9, 9000 Gent, Belgium \\ E-mail: attiliodifsc.usp.br, mendes@ifsc.usp.br
}

We report on the status of our study of gluon propagators and screening masses around the deconfining transition for pure SU(2) gauge theory in Landau gauge.

The XXIX International Symposium on Lattice Field Theory - Lattice 2011

July 10-16, 2011

Squaw Valley, Lake Tahoe, California

\footnotetext{
* Speaker.
} 


\section{Introduction}

Debye screening of the color charge, expected at high temperature, is signaled by screening masses/lengths that can in principle be obtained from the gluon propagator [1]. More specifically, chromoelectric (resp. chromomagnetic) screening will be related to the longitudinal (resp. transverse) gluon propagator computed at momenta with null temporal component (soft modes). In particular, we expect the real-space longitudinal propagator to fall off exponentially at long distances, defining a (real) electric screening mass, which can be calculated perturbatively to leading order. Also, according to the $3 \mathrm{~d}$ adjoint-Higgs picture for dimensional reduction, we expect the transverse propagator to show a confining behavior at finite temperature, in association with a nontrivial magnetic mass (see e.g. [2]). We note that these propagators are gauge-dependent quantities, and the (perturbative) prediction that the propagator poles should be gauge-independent must be checked, by considering different gauges.

Even though the nonzero- $T$ behavior just described has been verified for various gauges and established at high temperatures down to around twice the critical temperature $T_{c}[2,3]$, it is not clear how a screening mass would develop around $T_{c}$. At the same time, lattice studies of Landaugauge gluon propagators at finite temperature in pure $S U(2)$ and $S U(3)$ theory have observed a sharp peak in the infrared value of the electric propagator around the deconfinement temperature, suggesting an alternative order parameter for the QCD phase transition $[4,5,6,7,8]$. (Of course, a relevant question is, then, whether this singularity survives the inclusion of dynamical quarks in the theory [9].) In the following, we investigate the critical behavior of electric and magnetic gluon propagators and try to characterize the screening masses around the transition temperature $T_{c}$ by performing large-lattice simulations in pure $\mathrm{SU}(2)$ gauge theory. In particular, we use the knowledge gained in the study of the zero-temperature gluon propagator (see [10] for a review) to identify systematic effects in the infrared limit and to define temperature-dependent masses for the region around and below $T_{c}$. A more detailed analysis of our data will be presented elsewhere [11]. (Preliminary results were reported in $[12,13]$.)

\section{Results}

We have considered the pure $\mathrm{SU}(2)$ case, with a standard Wilson action. For our runs we employ a cold start, performing a projection on positive-Polyakov-loop configurations. Also, gauge fixing is done using stochastic overrelaxation and the gluon dressing functions are normalized to 1 at $2 \mathrm{GeV}$. We take $\beta$ values in the scaling region and lattice sizes ranging from $N_{s}=48$ to 192 and from $N_{t}=2$ to 16 lattice points, respectively along the spatial and along the temporal directions. Our (improved) procedure for determining the physical temperature $T$ is described in [13]. The momentum-space expressions for the transverse and longitudinal gluon propagators $D_{T}(p)$ and $D_{L}(p)$ can be found e.g. in [4]. We first describe our investigation of the critical behavior for transverse and longitudinal gluon propagators and then discuss our proposal for computing screening masses around $T_{c}$.

Our data for the transverse (magnetic) propagator $D_{T}(p)$ at the critical temperature are shown in Fig. 1. We clearly see the strong infrared suppression of the propagator, as expected, with a turnover at around $400 \mathrm{MeV}$. Regarding systematic errors, there are considerable finite-physical- 


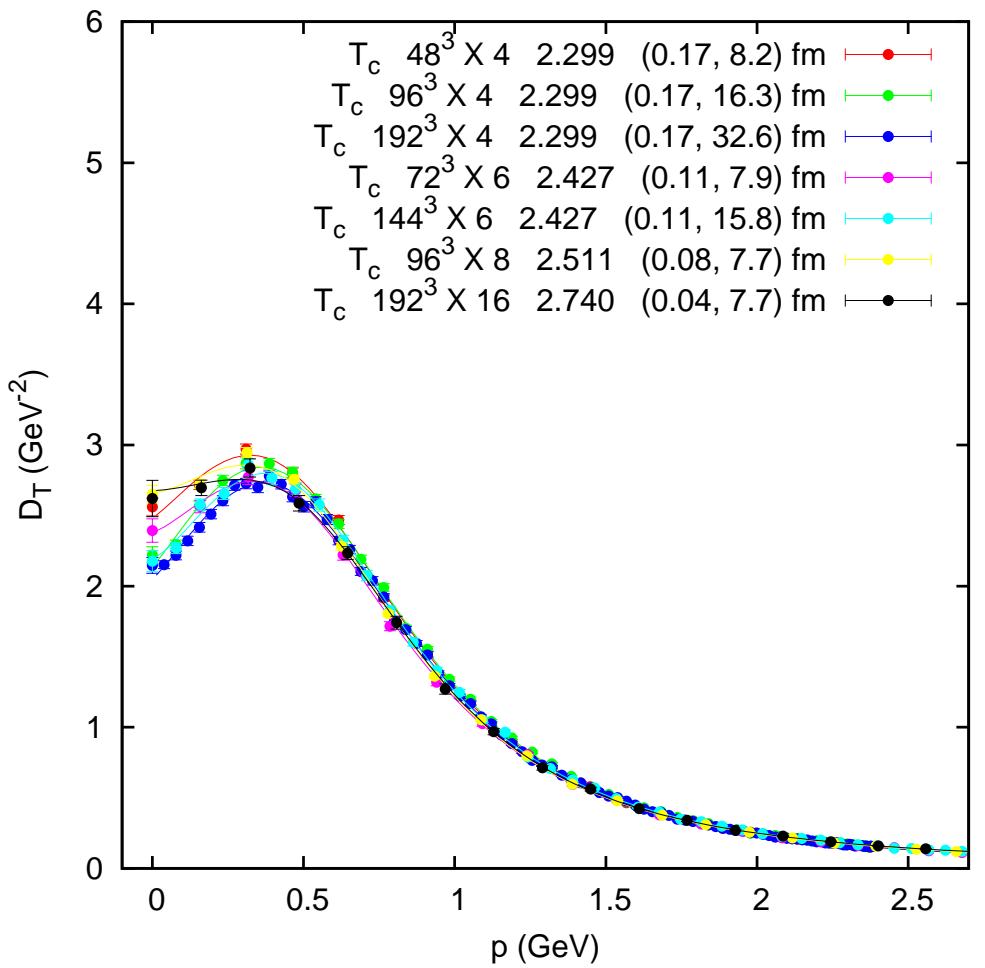

Figure 1: Transverse gluon propagator at $T_{c}$, for various choices of lattice size and $\beta$. Values for the temperature, $N_{s}^{3} \times N_{t}, \beta$, lattice spacing $a$ and spatial lattice size $L$ (both in $\mathrm{fm}$, in parentheses) are given in the plot labels. The solid lines are fits, described at the end of this section.

size effects, evidenced by the fact that the lattices with the smaller physical spatial size (the red, yellow, magenta and black curves) show different behavior from the remaining curves (green, blue and cyan). The nature of these effects is similar to what is observed at $T=0$ [10]. We mention that essentially the same features are seen for $D_{T}(p)$ at other temperatures, above and below $T_{c}$ [13].

The longitudinal (electric) propagator $D_{L}(p)$ at $T_{c}$ is shown in Fig. 2. We immediately see severe systematic effects for the smaller values of $N_{t}$. Let us note that our runs were initially planned under the assumption that a temporal extent $N_{t}=4$ might be sufficient to observe the infrared behavior of the propagators and our goal was, then, to increase $N_{s}$ significantly, to check for finite-size effects. As seen in Fig. 2, this assumption is not justified for the longitudinal propagator around the critical temperature, especially in the case of larger $N_{s}$. Indeed, as $N_{s}$ is doubled from 48 to 96 and then to 192 , we see that the infrared value of $D_{L}(p)$ changes drastically, resulting in a qualitatively different curve at $N_{s}=192$, apparently with a turnover in momentum. (Note that, in this case, the real-space longitudinal propagator manifestly violates reflection positivity.) We took this as an indication that our choice of $N_{t}=4$ was not valid and therefore considered larger values of $N_{t}$. We assume here that data points at $N_{t}=16$ are essentially free from systematic effects, since (as shown in [13]) the curves for temperatures around $T_{c}$ stabilize for $N_{t}>8$. As seen in the figure, we obtain in this way a different picture for the critical behavior of $D_{L}(p)$. It is interesting to note (see Fig. 2) that the $N_{s}$ effects at $T_{c}$ are significant for $N_{t}=6$ (with opposite sign with respect to the $N_{t}=4$ case) and are still present for $N_{t}=8$ (and maybe also for $N_{t}=16$ ). This is also true slightly 


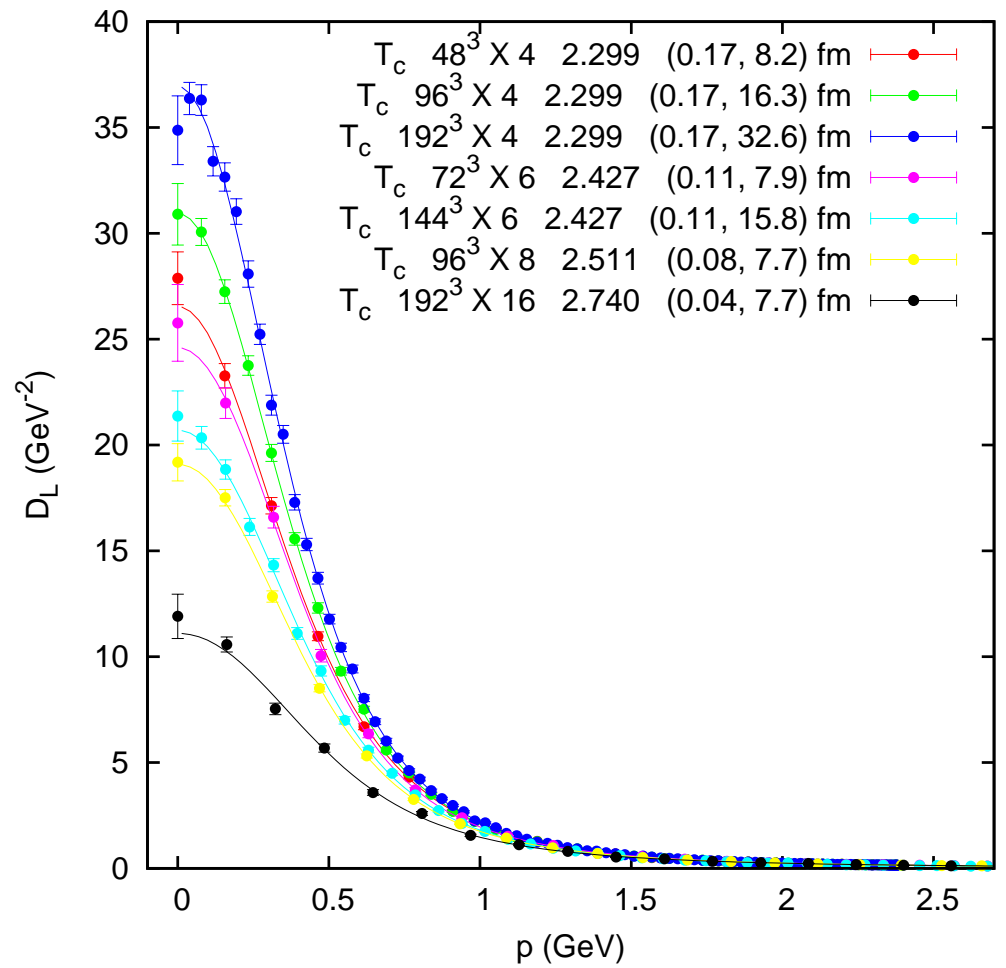

Figure 2: Longitudinal gluon propagator at and around $T_{c}$, for various choices of lattice size and $\beta$. Values for the temperature, $N_{s}^{3} \times N_{t}, \beta$, lattice spacing $a$ and spatial lattice size $L$ (both in $\mathrm{fm}$, in parentheses) are given in the plot labels. The solid lines are fits, described at the end of this section.

below $T_{c}$, but not immediately above $T_{c}[13]$.

In summary, the transverse propagator $D_{T}(p)$ shows significant finite-physical-size effects at $T_{c}$, while the longitudinal propagator $D_{L}(p)$ is subject to two sources of systematic errors for small $N_{t}$ : "pure" small- $N_{t}$ effects (associated with discretization errors) and strong dependence on the spatial lattice size $N_{s}$ at fixed $N_{t}$, when this value of $N_{t}$ is smaller than 16 . The latter effect was observed only at $T \lesssim T_{c}$, whereas the former is present in a wider range of temperatures around $T_{c}$ (see below). For all investigated values of the temperature, $D_{L}(p)$ seems to reach a plateau at small momentum $p$, while $D_{T}(p)$ is infrared-suppressed, with a turnover in momentum roughly around $350 \mathrm{MeV}$ for all $T \neq 0$.

Considering the infrared plateau in $D_{L}(p)$ - which we estimate here by $D_{L}(0)$ - as a function of temperature, the value observed at $T=0$ increases as the temperature is switched on, drops significantly for $T \gtrsim T_{c}$ and then shows a steady decrease. In Fig. 3, we show data for $D_{L}(0)$ for all our runs on the left-hand side, and for the region around $T_{c}$ on the right. We group together results from runs using the same value of $N_{t}$, and indicate them by the label "DL0_ $N_{t}$ ". The data points indicated with "sym" correspond to symmetric lattices, i.e. to the zero-temperature case. Note that results for different $N_{s}$ 's at fixed $N_{t}$ may not fall on top of each other, which gives us an indication of the systematic errors discussed above. These are especially serious for $N_{t}=4$ around $T_{c}$ (red points). We see that, surprisingly, the maximum value of $D_{L}(0)$ is not attained for $T=T_{c}-$ as might have appeared to be the case from the $N_{t}=4$ lattices only — and it does not correspond to a 

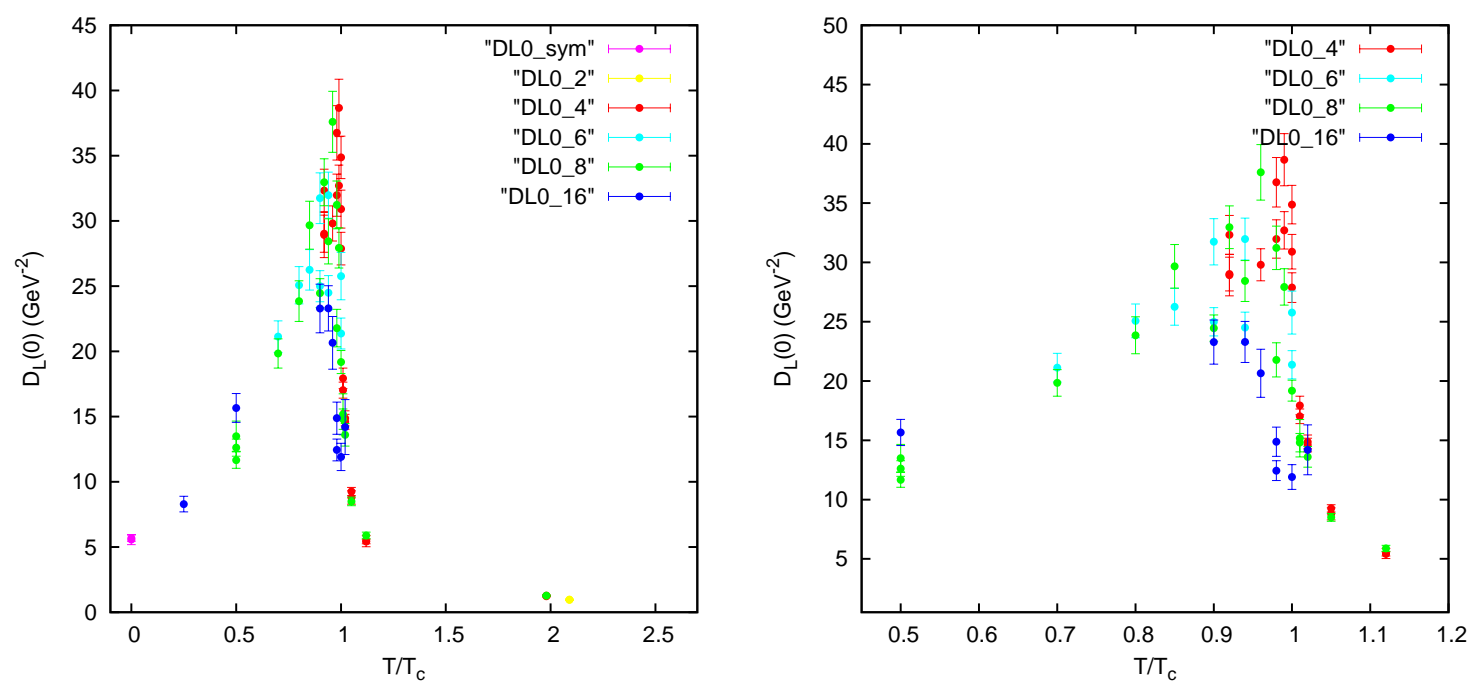

Figure 3: Infrared-plateau value for the longitudinal gluon propagator [estimated by $D_{L}(0)$ ] as a function of the temperature for the full range of $T / T_{c}$ values (left) and for the region around $T_{c}$ (right). Data points from runs at the same value of $N_{t}$ are grouped together and indicated by the label "DL0_N", where "sym" is used to indicate symmetric lattices (i.e. $T=0$ ).

flat curve from $0.5 T_{c}$ to $T_{c}$, as could be expected by looking only at these two temperatures. Rather, the maximum seems to lie at about $0.9 T_{c}$. Moreover, it clearly corresponds to a finite peak, which does not turn into a divergence as $N_{s}$ is increased at fixed $N_{t}$.

We have also looked at the real-space propagators. We find clear violation of reflection positivity for the transverse propagator at all temperatures. For the longitudinal propagator, positivity violation is observed unequivocally only at zero temperature and for a few cases around the critical region, in association with the severe systematic errors discussed above. For all other cases, there is no violation within errors. Also, we always observe an oscillatory behavior, indicative of a complex-mass pole. Typical curves for the longitudinal and transverse propagators in real space are shown (for $T=0.25 T_{c}$ ) in Fig. 4 .

We now address the problem of characterizing the screening masses around $T_{c}$. For all fits shown in Figs. 1 and 2 we used the same expression, namely a five-parameter fitting form of the Gribov-Stingl $[14,15]$ type $^{1}$

$$
D_{L, T}(p)=C \frac{1+d p^{2 \eta}}{\left(p^{2}+a\right)^{2}+b^{2}} .
$$

This form allows for two (complex-conjugate) poles, with masses $m^{2}=a \pm i b$, where $m=$ $m_{R}+i m_{I}$. The mass $m$ thus depends only on $a, b$ and not on the normalization $C$. The parameter $\eta$ should be 1 if the fitting form also describes the large-momenta region (from our infrared data we get $\eta \neq 1$ ). Recall that at high temperatures one usually defines the electric screening mass as the scale determining the exponential decrease of the real-space propagator at large distances, which is equivalent to $D_{L}(0)^{-1 / 2}$ in the case of a real pole. We therefore expect to observe $m_{I} \rightarrow 0$

\footnotetext{
${ }^{1}$ Note that, for given values of $a, b, d, \eta$, the global constant $C$ is fixed by the renormalization condition, so that there are only four free parameters in (2.1).
} 

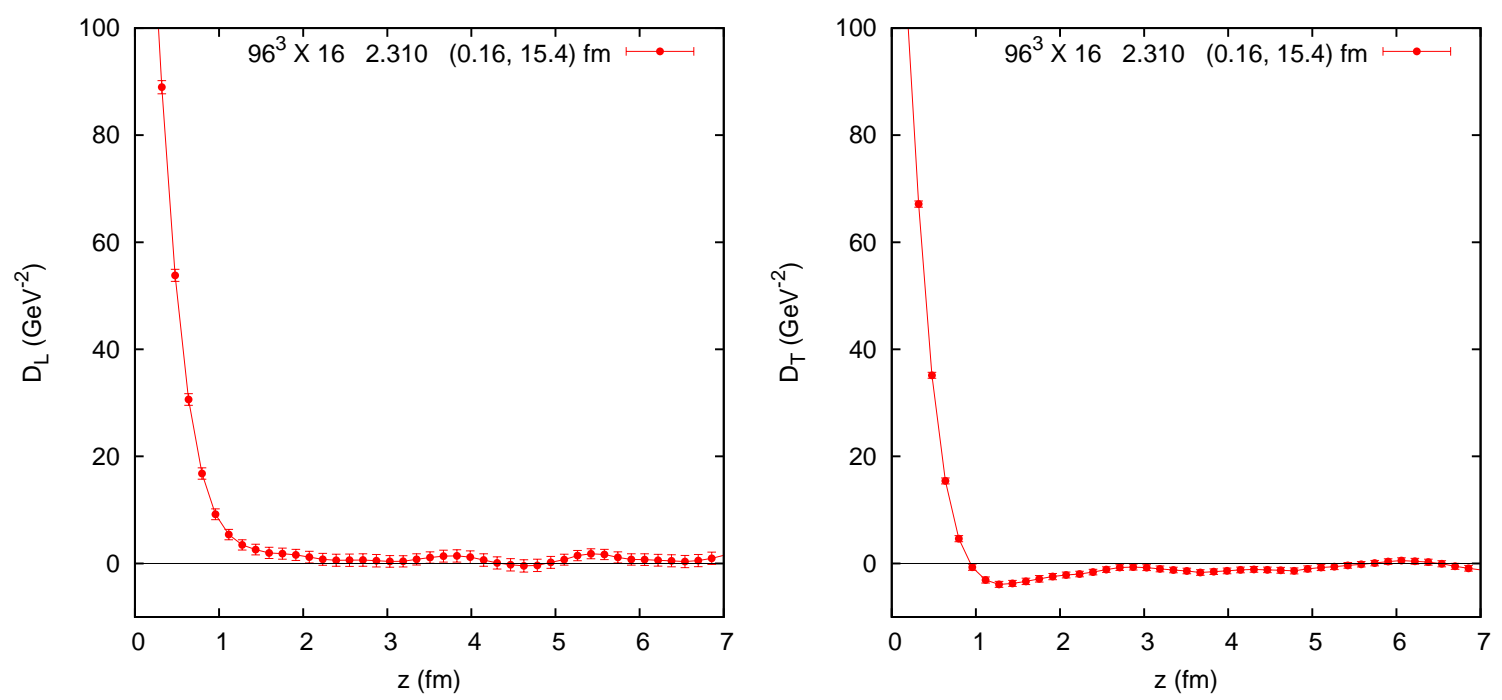

Figure 4: Longitudinal (left) and transverse (right) gluon propagator in real space for $T=0.25 T_{c}$. Values for $N_{s}^{3} \times N_{t}, \beta$, lattice spacing $a$ and spatial lattice size $L$ (both in $\mathrm{fm}$, in parentheses) are given in the plot labels. Note that the solid lines are not fits.

(i.e. $b \rightarrow 0$ ) for the longitudinal gluon propagator at high temperature. Note that, if the propagator has the above form, then the screening mass defined by $D_{L}(0)^{-1 / 2}=\sqrt{\left(a^{2}+b^{2}\right) / C}$ mixes the complex and imaginary masses $m_{R}$ and $m_{I}$ and depends on the (a priori arbitrary) normalization $C$.

We generally find good fits to the Gribov-Stingl form (including the full range of momenta), with nonzero real and imaginary parts of the pole masses in all cases. For the transverse propagator $D_{T}(p)$, the masses $m_{R}$ and $m_{I}$ are of comparable size (around 0.6 and $0.4 \mathrm{GeV}$ respectively). The same holds for $D_{L}(p)$, but in this case the relative size of the imaginary mass seems to decrease with increasing temperature. A detailed discussion of the associated masses $m_{R}, m_{I}$ is postponed to a forthcoming study [11], as we are presently considering variants of the above fitting form inspired by zero-temperature studies. Indeed, the use of a Gribov-Stingl form is motivated by the behavior of the gluon propagator at $T=0$, where this type of expression has been shown to describe well the data in three space-time dimensions [16]. Recently, in [17], various fitting forms of this type were used to describe large-lattice data for the $3 \mathrm{~d}$ and $4 \mathrm{~d}$ gluon propagator at $T=0$. Noting that the $3 \mathrm{~d}$ case may be considered as the $T \rightarrow \infty$ limit of the $4 \mathrm{~d}$ case, we propose to interpolate the $3 \mathrm{~d}$ and $4 \mathrm{~d}$ zero-temperature forms to describe our finite- $T$ data in $4 \mathrm{~d}$.

\section{Conclusions}

We study the longitudinal (electric) and transverse (magnetic) gluon propagators in momentum space, proposing the calculation of screening masses through an Ansatz from the zero-temperature case. Going from zero to nonzero temperature, we see that the electric propagator $D_{L}(p)$ is enhanced, with an apparent plateau value in the infrared, while the magnetic propagator $D_{T}(p)$ gets progressively more infrared-suppressed, with a clear turnover in momentum at all nonzero temperatures considered. Severe systematic effects are observed for the electric propagator around $T_{c}$, suggesting that lattices of temporal extent $N_{t}>8$ are needed for this study. Once these errors 
are removed, the data support a finite maximum (located at about $0.9 T_{c}$ ) for the infrared value of $D_{L}(p)$.

\section{Acknowledgements}

The authors thank agencies FAPESP and CNPq for financial support. A. Cucchieri also acknowledges financial support from the Special Research Fund of Ghent University (BOF UGent). Our simulations were performed on the new CPU/GPU cluster at IFSC-USP (obtained through a FAPESP grant).

\section{References}

[1] D. J. Gross, R. D. Pisarski, L. G. Yaffe, Rev. Mod. Phys. 53, 43 (1981).

[2] A. Cucchieri, F. Karsch, P. Petreczky, Phys. Rev. D 64, 036001 (2001).

[3] U. M. Heller, F. Karsch, J. Rank, Phys. Rev. D 57, 1438 (1998); A. Cucchieri, F. Karsch, P. Petreczky, Phys. Lett. B 497, 80 (2001).

[4] A. Cucchieri, A. Maas, T. Mendes, Phys. Rev. D 75, 076003 (2007).

[5] C. S. Fischer, A. Maas, J. A. Muller, Eur. Phys. J. C 68, 165 (2010).

[6] V. G. Bornyakov and V. K. Mitrjushkin, Phys. Rev. D 84, 094503 (2011) [arXiv:1011.4790 [hep-lat]].

[7] R. Aouane, V. Bornyakov, E. -M. Ilgenfritz, V. Mitrjushkin, M. Muller-Preussker and A. Sternbeck, arXiv:1108.1735 [hep-lat].

[8] A. Maas, J. M. Pawlowski, L. von Smekal and D. Spielmann, arXiv:1110.6340 [hep-lat].

[9] V. G. Bornyakov, V. K. Mitrjushkin, arXiv:1103.0442 [hep-lat].

[10] A. Cucchieri and T. Mendes, PoS QCD -TNT09, 026 (2009) [arXiv:1001.2584 [hep-lat]].

[11] A. Cucchieri, T. Mendes, in preparation.

[12] A. Cucchieri and T. Mendes, PoS LATTICE 2010, 280 (2010) [arXiv:1101.4537 [hep-lat]].

[13] A. Cucchieri and T. Mendes, PoS FACESQCD , 007 (2010) [arXiv:1105.0176 [hep-lat]].

[14] M. Stingl, Phys. Rev. D 34, 3863 (1986) [Erratum-ibid. D 36, 651 (1987)].

[15] M. Stingl, Z. Phys. A 353, 423 (1996) [hep-th/9502157].

[16] A. Cucchieri, T. Mendes and A. R. Taurines, Phys. Rev. D 67, 091502 (2003) [hep-lat/0302022].

[17] A. Cucchieri, D. Dudal, T. Mendes and N. Vandersickel, arXiv:1111.2327 [hep-lat]. 